\title{
经鼻间歇正压通气用于新生儿呼吸莬迫综合征的临床价 值分析
}

\author{
盛亚勤* \\ 襄城县人民医院新生儿监护室, 河南 461700
}

摘 要: 目的：探讨分析对新生儿呼吸客迫综合征患儿使用经鼻间歇正压通气的治疗效果。方法: 本次研究对象 均选自本院2019年3月到2021年3月期间收治的新生儿呼吸客迫综合征患儿共108例, 按照双盲法对患儿分组, 设定其 中54例为参照组采用经鼻持续正压通气进行治疗, 其余54例作为研究组采用经鼻间歇正压通气进行治疗, 观察对两组 的治疗效果。结果: 比较两组的治疗成功率, 研究组高于参照组 $(P<0.05)$; 比较两组的吸氧时间以及住院时间, 研究组均少于参照组 $(P<0.05)$; 比较两组的并发症发生率, 研究组低于参照组 $(P<0.05)$ 。结论: 根据本次研究 的结果可以确认，对新生儿呼吸容迫综合征患儿使用经鼻间歇正压通气的治疗效果更为理想，还可以有效避免患儿出 现并发症问题，从而促进患儿的病情恢复，有必要在临床上广泛应用。

关键词：新生儿呼吸窘迫综合征；经鼻间歇正压通气；经鼻持续正压通气；治疗效果；并发症；对比分析

\section{一、前言}

新生儿呼吸莬迫综合征属于临床上比较常见的一种新生儿疾病, 主要出现于不足35周胎龄的早产儿。这主要是因 为早产儿由于娩出时间过早, 其肺组织尚未发育完全, 缺乏肺表面活性物质有关。患儿在发病后, 将会出现进行性呼 吸困难以及肺不张等多种症状，如果没有对患儿采取及时有效的治疗措施，将会对患儿的生命造成直接的威胁 ${ }^{[1]}$ 。在 对患儿治疗时, 主要采取的治疗手段为机械通气治疗, 可以帮助患儿维持正常的呼吸, 避免患儿出现死亡的严重。目 前临床上常用的机械通气方法为经鼻持续正压通气，该治疗方法虽然可以取得一定的治疗效果，但是容易引发患儿出 现多种并发症。因此，本文旨在探讨分析对新生儿呼吸客迫综合征患儿使用经鼻间歇正压通气的治疗效果。

\section{二、资料和方法}

(一) 一般资料

本次研究对象均选自本院2019年3月到2021年3月期间收治的新生儿呼吸客迫综合征患儿共108例, 按照双盲法对 患儿分组。本次研究已经我院伦理委员会批准, 全部患儿及其监护人均对本次研究知情, 并且患儿的监护人也已签署 知情同意书。设定其中 54例为参照组采用经鼻持续正压通气进行治疗, 其余 54 例作为研究组采用经鼻间歇正压通气进 行治疗。研究组中，男患儿29例，女患儿25例为，出生时间为 1 小时到 22 小时，平均出生时间为 $10.3(s=1.2)$ 小时; 体重为 1.21 千克到 2.26 千克, 平均体重为 $1.7(s=0.3)$ 千克。参照组中, 男患儿 29 例, 女患儿 25 例为, 出生时间为 1 小 时到 22 小时, 平均出生时间为 $10.6(s=1.5)$ 小时; 体重为 1.26 千克到 2.35 千克, 平均体重为 $1.8(s=0.4)$ 千克。对比 两组患儿的常规资料不存在明显差异 $(P>0.05)$ 。

(二) 方法

两组患儿在发病后，均对其给予肺泡表面活性物质治疗，并采取基础护理。

1. 参照组患儿

采用经鼻持续正压通气进行治疗, 治疗方法为通过双鼻塞密闭环路方式对患儿开展经鼻持续正压通气，持续向患 儿的气道施加一定程度的正压力, 需要保证患儿的呼吸末正压维持在5厘米水柱到6厘米水柱之间, 吸气峰压维持在 15 厘米水柱到 20 厘米水柱之间，吸气时间在 0.3 秒到 0.5 秒之间，呼吸频率在每分钟 25 次到 50 次之间，吸人氧浓度在 $30 \%$ 到60\%之间。

“通讯作者：盛亚勤，1983年8月，女，汉族，河南许昌人，就职于襄城县人民医院新生儿重症监护室，主治医 师, 本科学历。研究方向: 儿科。 


\section{2. 研究组患儿}

采用经鼻间歇正压通气进行治疗，治疗方法为通过双鼻塞密闭环路方式对患儿开展经鼻间歇正压通气，机械提高 患儿咽喉部的压力, 从而提高患儿的上呼吸道压力。需要保证患儿的呼吸末正压维持在5厘米水柱到6厘米水柱之间, 吸气峰压维持在 15 厘米水柱到 20 厘米水柱之间, 吸气时间在 0.3 秒到 0.5 秒之间, 呼吸频率在每分钟 25 次到 50 次之间, 吸人氧浓度在 $30 \%$ 到 $60 \%$ 之间。

两组患儿在接受治疗时, 需要密切观察患儿的血气之间以及临床症状, 对呼吸机参数进行适当的调整。如果患儿 的吸气峰压超过 20 厘米水柱、呼吸频率超过每分钟 50 次或者经皮氧饱和度低于 $90 \%$ 亦或者吸氧浓度超过 $60 \%$, 则表明 患儿的呼吸客迫症状加重, 需要及时转为气管插管机械通气, 控制患儿的病情。在患儿的临床症状得到控制后, 再合 理调整相关参数，并在患儿病情得到控制后，将呼吸机撤除。

\section{(三) 观察指标}

在两组患儿经过治疗后，根据对患儿的治疗结果，评判对患儿的治疗是否成功。

1. 成功

在对患儿治疗后, 其呼吸困难、躁动以及哭闹等症状基本消失，未见明显的发细以及三凹征等情况。患儿呼吸频 率恢复正常，可以将呼吸机撤除，不再需要机械通气。

2. 失败

患儿在经过治疗后, 其病情未见明显改善, 患儿无法脱离呼吸机或者是出现死亡的严重后果。同时, 观察患儿在接 受治疗过程中的并发症（呼吸道黏膜损伤。肺出血、肺部感染）发生情况，并且统计患儿的吸氧时间以及住院时间 ${ }^{[2]}$ 。

(四) 统计学分析

通过SPSS 22.0 统计学软件进行分析, 用百分比表示计数资料, 行卡方检验; 对计量资料行 $t$ 检验, 用均数 \pm 标准 差进行表示。 $P<0.05$ 表明对比有差异。

\section{三、结果}

(一) 比较两组的治疗成功率

比较两组的治疗成功率, 研究组高于参照组 $(P<0.05)$ 。详情如表1所示。

表1 比较两组的治疗成功率 $[n /(\%)]$

\begin{tabular}{ccccc}
\hline 组别 & $\mathrm{n}$ & 成功 & 失败 & 成功率 \\
\hline 参照组 & 54 & 38 & 16 & 70.37 \\
研究组 & 54 & 49 & 5 & 90.74 \\
$x^{2}$ & $/$ & $/$ & $/$ & 5.982 \\
$P$ & $/$ & $/$ & $/$ & 0.014 \\
\hline
\end{tabular}

(二) 比较两组的并发症发生率

比较两组的并发症发生率, 研究组低于参照组 $(P<0.05)$ 。详情如表2所示。

表2 比较两组的并发症发生率 $[n /(\%)]$

\begin{tabular}{cccccc}
\hline 组别 & $n$ & 肺部感染 & 呼吸道黏膜损伤 & 肺出血 & 总发生率 \\
\hline 参照组 & 54 & 6 & 4 & 5 & 27.78 \\
研究组 & 54 & 1 & 1 & 1 & 5.56 \\
$x^{2}$ & $/$ & $/$ & $/$ & $/$ & 9.600 \\
$P$ & $/$ & $/$ & $/$ & $/$ & 0.002 \\
\hline
\end{tabular}

（三）比较两组的吸氧时间以及住院时间

研究组的吸氧时间为 $(2.2 \pm 0.4)$ 天、住院时间为 $(5.8 \pm 0.6)$ 天，参照组的吸氧时间为 $(4.9 \pm 0.5)$ 天、住院时 间为 $(8.3 \pm 0.6)$ 天。比较两组的吸氧时间以及住院时间, 研究组均少于参照组 $(t=28.547, P=0.000 ; t=19.523, P$ $=0.000)$ 。

四、讨论

新生儿呼吸客迫综合征患儿在发病后, 会出现明显的呼吸困难症状, 如果没有采取及时有效的控制措施, 该症状 
还会持续加重, 并最终导致患儿死亡。该病的发生与患儿的胎龄有着密切的关系, 患儿的胎龄越小发病的概率也就 越大。随着现代人们思维方式的转变, 使得剖宫产在临床上越发常见, 这也在一定程度上提高了该病的发生率。通常 新生儿在出生后就可以有正常的呼吸, 如果新生儿在出生6小时后, 就出现呼气性呻吟以及进行性加重的呼吸困难症 状, 则可以确认患儿为新生儿呼吸客迫综合征 ${ }^{[3]}$ 。在确认患儿发病后, 纠正其缺氧状态以及改善其通气状态是关键, 需要使患儿获取充足的氧气, 才可以避免其出现严重的后果。目前, 临床上通常对患儿采取机械通气技术进行治疗, 但是容易导致患儿出现呼吸机相关性肺炎等并发症, 影响患儿的愈后效果。所以, 主张对患儿采取无创机械通气治 疗，这不仅可以减少患儿的用氧时间，还可以避免对患儿气管插管，从而更好地改善患儿的病情。

现如今在临床上的常用无创机械通气方法为经鼻持续气道正压通气以及经鼻间歇正压通气两种方法, 前者将会对 患儿的气道持续产生压力, 这样可以改善患儿的呼吸做功, 降低患儿呼吸时对肺泡表面活性物质的消耗, 从而保证 其气道保持在较好的扩张程度上, 增强患儿肺的顺应性, 避免患儿出现肺泡塌陷的问题 ${ }^{[4]}$ 。但是, 患儿在治疗的过程 中, 很容易会出现多种并发症问题, 引发患儿出现频繁呼吸暂停以及自主呼吸减弱等问题, 并且有相当一部分患儿在 治疗后, 还需要采用气管插管机械通气 ${ }^{[5]}$ 。后者则可以间歇性地提高患儿的咽喉压力, 还能够增强患儿的潮气量, 这 样就能够使患儿的气道处于平均气压的状态, 从而切实的改善患儿的肺活量, 促使患儿出现萎缩的肺泡重新恢复张 力, 提高患儿肺部的气体交换功能以及氧和功能。并且, 不管患儿是否存在自主呼吸, 都可以有效增强患儿的平均气 道压, 有着十分理想的呼吸支持作用, 避免患儿出现呼吸暂停的问题, 降低气管插管率, 防止对患儿的肺部造成损 伤, 提高对患儿治疗的安全性和有效性。同时, 患儿的肺部功能恢复速度更加理想, 能够帮助患儿更快地摆脱呼吸 机，避免患儿出现严重的后果 ${ }^{[6]}$ 。

本次研究结果显示: 比较两组的治疗成功率, 研究组高于参照组 $(P<0.05)$ 。这说明对患儿采用经鼻间歇正压 通气治疗可以有效提高治疗成功率; 比较两组的吸氧时间以及住院时间, 研究组均少于参照组 $(P<0.05)$ 。这说明 对患儿采用经鼻间歇正压通气治疗可以显著降低其住院时间以及吸氧时间; 比较两组的并发症发生率, 研究组低于参 照组 $(P<0.05)$ 。这说明对患儿采用经鼻间歇正压通气治疗可以有效预防并发症。

综上所述, 对新生儿呼吸客迫综合征患儿使用经鼻间歇正压通气的治疗效果更为理想, 还可以有效避免患儿出现 并发症问题，从而促进患儿的病情恢复，有必要在临床上广泛应用。

\section{参考文献:}

[1]李华.经鼻同步间歇正压通气治疗新生儿呼吸蹇迫综合征的临床价值研究[J].中外医学研究, 2019,17(7):54-55.

[2]简伟华,袁贯平.经鼻间歇正压通气应用于新生儿呼吸蹇迫综合征治疗的临床效果[J]. 黑龙江中医药, 2019,v.48; No.306(4):36-37.

[3]陈秋萍,覃广萍, 罗勤, 经鼻间歇正压通气联合猪肺磷脂注射液治疗新生儿呼吸蹇迫综合征的临床效果 [J].中国现代 医生, 2019,57(17):70-72.

[4]申英杰, 尉进茜, 詹实娜, 赵辉, 赵天娇, 张倩. 经鼻间歇正压通气与经鼻持续气道正压通气治疗早产儿呼吸寒迫综合 征的疗效比较[J].世界临床医学, 2019,13(2):76-77.

[5]邓婉, 周玉娥, 杨严政, 廖桂.间歇俯卧位通气在新生儿呼吸蹇迫综合征中的应用效果研究[J].昆明医科大学学报, 2021,42(6):1-4.

[6]洪柳青.经鼻间歇正压通气在新生儿呼吸蹇迫综合征中的应用价值[J].医学理论与实践, 2019,32(2):246-248. 